\title{
The equivalence of convex and concave transport cost in a circular spatial model with and without zoning*
}

\author{
La equivalencia del coste de transporte cóncavo y convexo en un mercado \\ circular con y sin zonificación**
}

\author{
HAMid HaMOUd

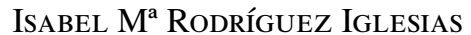 \\ Marcos Sanz Martín-Bustamante***
}

\begin{abstract}
This article depicts a location game in a circular market. The equivalence results between a convex and a concave transport cost are reexamined by assuming an arbitrary length. In contrast to previous research the solution found shows that the equivalence relationship depends on the space length. Furthermore, the analysis is extended to a circular model with unitary length and zoning. In this case equivalence does not hold. Moreover, non-existence of equilibrium is shown under strictly linear quadratic functions. Surprisingly, equilibrium exists for a concave quadratic function but not for a convex quadratic function.
\end{abstract}

Key words: Convexity, concavity, spatial competition, circular model, transport costs, regulator.

JEL Classification: C72, D43, L13, R38.

\section{Resumen}

Este artículo describe un juego de localización en un mercado circular. Se reexamina el resultado de equivalencia entre los costes de transporte cóncavos y convexos asumiendo una longitud arbitraria. Al contrario que en investigaciones previas, se encuentra una solución que muestra que la relación de equivalencia depende de la longitud del espacio. Asimismo se extiende el análisis a un modelo circular con una longitud unitaria y zonificación. En este caso no se cumple la equivalencia. Además se demuestra la existencia de equilibrio para funciones estrictamente cuadrático-lineales. Sorprendentemente se produce

* We thank the anonymous reviewers and the editor of Estudios de Economía for helpful comments which have considerably improved the original manuscript.

** Este artículo ha recibido apoyo del proyecto del Ministerio de Ciencia y Tecnología ECO2012-322 y del Proyecto RSC de UNIR Research.

*** Universidad Rey Juan Carlos de Madrid. Paseo Artilleros s/n. Madrid 28032. hamid. hamoudi@urjc.es, isabel.rodriguez@urjc.es, marcos.sanz.martinbustamente@urjc.es 
el equilibrio para una función cuadrática cóncava pero no para una función cuadrática convexa.

Palabras clave: Convexidad, concavidad, competencia espacial, modelo circular, costes de transporte, regulador.

JEL Classification: C72, D43, L13, R38.

\section{INTRODUCTION}

Spatial competition models consider consumers and firms distributed along a line or a circle. Both spatial configurations are important. The linear model à la Hotelling represents situations where there are exogenous endpoints; leading the distinct locations to be differentiated in terms of their relative positions to the borders- thus the market along the line is not perfectly homogeneous. Namely, the linear market assumption allows determination of location patterns which arise from various market characteristics, due to its intrinsic heterogeneity. In contrast, the circular city à la Salop case is perfectly homogeneous since location patterns are differentiated in terms of the firms' relative locations to each other. Therefore, the circular framework is more appropriate for certain real-life situations. Typical examples are traffic-jammed cities with large shopping malls located on the outskirts, along the circular belt-way, to avoid downtown traffic for shoppers, competing television networks when choosing time slots for their shows, airlines deciding on arrival and departure times for their flights... etc. In terms of product specification, the linear representation is often preferred because it applies to single-peaked consumer preferences, whereas no such analogy is available for the circular model.

These models usually assume a convex ${ }^{1}$ type of transport function and very often ignore concave ${ }^{2}$ transport cost functions. It is well known that choosing the type of costs depends on what is included in the concept of distance (itinerary, time, degree of buying advantages). However, there exists no clear evidence in favor of one or another transport cost in spatial competition models.

Given the nature of our investigation, the focus will be on the circular space. The first part builds on the model from De Frutos et al. (1999) in order to analyse the equivalence game induced by a convex and a concave linear quadratic transport cost function in a circular market with an arbitrary length. In this context, it is shown that the equivalence result crucially depends on the length of the market. This outcome renders the analysis for the unit length a particular case from the general conclusions presented here. On the other hand, it can be taken as a precise rule of thumb for any urban city planner dealing with the choice on the appropriate side of a market.

\footnotetext{
See D’Aspremont et al. (1979), Gabszewics et al. (1986), Anderson (1986), Hamoudi et al. (2011) among others.

2 De Frutos et al. (1999, 2002), Hamoudi et al. (2005), Matsumura (2006).
} 
The second part extends the analysis by introducing a regulator. More specifically, the regulator restricts the location space of the agents in a circular market. Firms and consumers locate in the unit circle in separated areas: commercial and residential.

The study of regulation in this type of models contributes to urban industrial policy. For example, when choosing areas to locate new shops firms are often faced with political restrictions such as: green zone preservation, existing hospitals, or residential areas. In turn, zoning becomes an important instrument to plan rational space occupation by choosing optimal specific areas for the location of firms. The ban on the sale of all alcohol in residential areas constitutes a typical example of this kind of policy. The reasons for zoning in this case are twofold: alcohol consumption is associated with the negative effects of delinquency (Carpenter, 2007) and greater distance between consumers and sellers discourages consumption (Cook et al. 2000). A second paradigmatic example is the exclusion of gasoline stations from residential areas (Netz et al. 2002).

Initially, it is shown that the equivalence result cannot be extended to the model under zoning. Then, a proof is provided to demonstrate that price equilibrium is not guaranteed for every possible location of firms under convex/ concave strictly linear quadratic transportation costs. Unexpectedly, the convex quadratic transport cost function cannot deliver price equilibrium for any location of firms. Thus, the equilibrium properties associated with this function are not as robust as many researchers suggest, D’Aspremont et al. (1979), Anderson (1986), (1988), Gabszewicz et al. (1986), Lambertini (1994), Böckem (1994), Tabuchi et al. (1995), Junichiro et al. (2004) and Brenner (2005). Notably, the concave quadratic transport cost function yields perfect price-location equilibrium. Moreover, this equilibrium is unique and characterized by maximum differentiation (dispersion).

This article is structured as follows: section two introduces the standard model and studies the equivalence of the games induced by a convex and a concave transport cost function. In section three the regulated circular model is analysed in order to verify the equivalence of the games and concludes by seeking for the existence of Nash equilibrium in prices. Section four presents the main conclusions.

\section{ReVisiting The EquivalenCe RESUlt}

\subsection{The model}

In the following example, the basic assumptions from Hotelling's model (1929) are maintained except for the fact that the market considered is circular with a length of $2 L$. There are two firms which produce a homogeneous good, with zero production costs. Consumers are uniformly distributed along a circular city. Each consumer is constrained to buy one unit of good. Firm 1 locates at $x_{1} \in[0, L]$ and sells the product at price $p_{1}$. Similarly, firm 2 locates at $x_{2} \in[0, L]$ such that $x_{1} \leq x_{2}$ and sells the product at $p_{2}$. The consumer location denoted by $\alpha$ corresponds to a point in the circle and is identified with numbers in $[0,2 \mathrm{~L}]$. The most southern location is taken as 0 and values increase counter clockwise. Thus, the most southern location is considered both 0 and $2 \mathrm{~L}$. The distance be- 
tween the consumer and the firm is defined by $d_{i}(\alpha)=\left|\alpha-x_{\mathrm{i}}\right|$. Sellers follow an f.o.b price policy and consumers must travel to buy one unity of good and bear transport costs, denoted by $C\left(d_{i}\right)$.

\section{FIGURE 1}

THE CIRCULAR MODEL MARKET

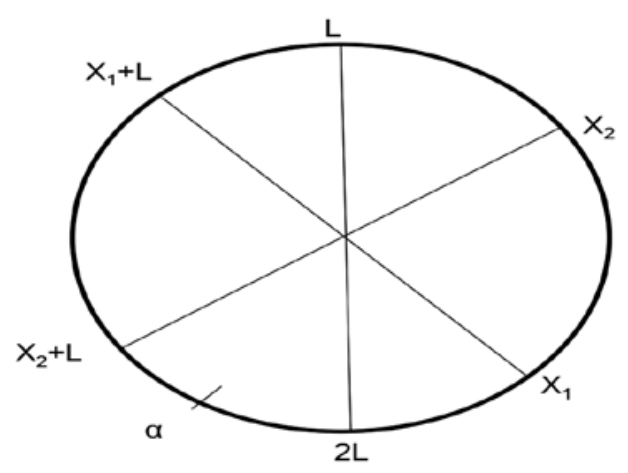

Consumers can travel along the whole circle and will always take the direction implying the shorter distance to the chosen firm. Since the product is homogeneous, a consumer purchases from the seller with the lowest delivered price which consists of mill price plus transport cost. The indifferent consumer is defined as the consumer who faces the same full price from both firms so that: $p_{1}+C\left(d_{1}\right)=p_{2}+C\left(d_{2}\right)$.

The transport cost is assumed to be non-negative, continuous and increasing in distance with zero cost when travelling zero distance. This function can be convex or concave.

The main purpose from here on is to demonstrate that the game induced by a convex/concave function is equivalent to the game induced by a concave/ convex function.

\subsection{Results}

In order to achieve the above goal the following lemma is stated:

\section{Lemma 1:}

Given continuous and differentiable a transport cost function $F(d)$, where $d \in[0, L]$, another transport cost function can be found $H\left(d^{\prime}\right)$ such that: $d=L-d$, $H\left(d^{\prime}\right)=F(L)-F\left(d^{\prime}\right)$.

Proof: The length of the circular market in the model considered is equal to $2 \mathrm{~L}$. A consumer can travel a maximum distance of $\mathrm{L}$ involving a transport cost of 
$C(L)$ whereas the minimum distance is zero with zero transport cost associated. Departing from an initial coordinate system $\mathrm{S}_{1}$ with origin $\mathrm{O}=(0,0)$ and axes $d, C(d)$; a second reference system $\mathrm{S}_{2}$ can be defined by shifting the origin $\mathrm{O}$ to $\mathrm{O}^{\prime}=(L, C(L))$ and rotating 180 degrees. All pairs $(d, C(d))$ from $\mathrm{S}_{1}$ can then be represented in $\mathrm{S}_{2}$ under the following change of variable: $d^{\prime}=L-d, H\left(d^{\prime}\right)=F(L)$ $-F(d)$. If $d$ ' is substituted by $L-d$ into $H, F(d)=H(L)-H(L-d)$ is obtained.

Note that $H(0)=F(0)=0$ and $H(L)=F(L)$. If $\partial^{2} F(d) / \partial^{2} d \geq 0$, i.e. $F(d)$ is a convex function then $H\left(d^{\prime}\right)$ is a concave function $\left(\partial^{2} H\left(d^{\prime}\right) / \partial^{2} d^{\prime} \leq 0\right)$. Contrary, if $\mathrm{F}(\mathrm{d})$ is concave then $\mathrm{H}\left(\mathrm{d}^{\prime}\right)$ is convex.

The central proposition of this section can be stated now:

\section{Proposition 1:}

In a circular market of length $2 L$, the location-then-price game induced by a transport cost function $F$ is fully equivalent to the game induced by a transport cost function $H$, where $H(d)=F(L)-F(L-d)(1)$.

Proof: The result follows from lemma 1 above. By relying on definition 1; lemma 1; the proof of theorem 2 and corollary 3 in De Frutos et al. (2002) it can be proved straightforward.

This result constitutes an extension of De Frutos et al. (2002) since it provides a generalization for the equivalence relation by showing how it depends on the market length.

To illustrate the relationship between convex and concave transport cost some functions from the linear quadratic family can be examined:

The convex function is given by, $C^{+}\left(d_{i}\right)=a^{+} d_{i}+b^{+} d_{i}^{2}$, where $a^{+} \geq 0, b^{+} \geq 0$ and the concave function is represented by, $C^{-}\left(d_{i}\right)=a^{-} d_{i}-b^{-} d_{i}^{2}$, where $a>0, b \geq 0$. It is assumed that $\mathrm{L} \leq\left(\mathrm{a}^{-} / 2 \mathrm{~b}^{-}\right)$to assure that the concave transport cost function is increasing in the whole market.

\section{Corollary 1:}

The location-then-price game induced by convex and linear quadratic transport cost function, $C^{+}\left(d_{i}\right)$, is fully equivalent to the game induced by concave and linear quadratic transport cost function, $C^{-}\left(d_{i}\right)$ if and only if the length of the market in this is equal to $2 L=\left(a^{-}-a^{+}\right) / b^{-}$and $b^{+}=b^{-}$

Proof: Via the relation (1) between convex and concave cost, a relationship between $a^{+}, b^{+}$and $a^{-}, b^{-}$can now be derived and represented as: $a^{+}=a^{-}-2 b^{-} L, \quad b^{+}=b^{-}$(2). Then relationship between parameters $\mathrm{L}$ and $a^{+}, a^{-}, b^{-}$can be deducted from here.

\section{Remarks:}

Note that this result provides a specific relationship between the weights attached to the linear and non linear parts of the transport cost functions and the length of the circular market considered. This means that any existing public or private authority could take into account considerations about the weight of the parameters in the transport cost functions when deciding on the ideal size of 
the space market. In this respect, the result provided here allows a city planner to be indifferent about the type of transport cost function.

For the case of a unitary length circular market, i.e. $2 \mathrm{~L}=1$ under convex linear quadratic transportation De Frutos et al. (1999, proposition 1) show that there exists a subgame perfect price-location equilibrium iff $a^{+}=0$. They then prove that the game induced by the concave and linear quadratic transport cost function $C^{-}\left(d_{i}\right)=b^{-}\left(d_{i}-d_{i}^{2}\right)$, i.e. $a^{-}=b^{-}$, has a unique subgame perfect equilibrium. More importantly for comparison purposes, the above function, $C^{-}\left(d_{i}\right)=b^{-}\left(d_{i}-d_{i}^{2}\right)$, yields equilibrium in the circular market only if $\mathrm{L}=1 / 2$. At this point, it is worth noting that the result from De Frutos et al. remains as a particular case for $L=1 / 2$. If any other value of $L$ is considered their function does not lead to equilibrium. However, by using corollary 1 we show that the function, $C^{-}\left(d_{i}\right)=b^{-}\left(2 L d_{i}-d_{i}^{2}\right)$ leads to equilibrium for any value of $\mathrm{L}$, therefore, generalizing results from De Frutos et al. For the above concave function the equilibrium pattern remains similar in terms of maximum differentiation which corresponds to spatial dispersion ${ }^{3}$.

\section{Circular market with zoning}

\subsection{Model}

In this section a duopoly location model is analyzed in a circular market under zoning regulation. An authority divides a circular city of length 1 into two distinct parts, a commercial area of length $v$, such that $0 \leq v \leq 1 / 2$ and a residential area of length $1-v$.

The regulated circular model as represented in Figure 2 is shown below:

FIGURE 2

REGULATED CIRCULAR MODEL

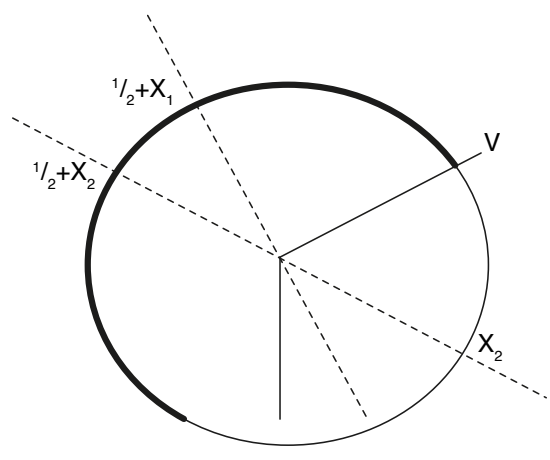

3 Simple calculations, similar to those in De Frutos et al. (1999), show that for the above function a unique perfect-location equilibrium for the convex and concave case and any value of $\mathrm{L}$ exists, which is given by, $x_{1}=0, x_{2}=L, \quad p_{1}=p_{2}=b^{+} / 4$. 
Two firms located at $x_{1}$ and $x_{2}$, with $x_{1}, x_{2} \in[0, v]$ and $x_{1} \leq x_{2}$ charge mill prices $p_{1}$ and $p_{2}$. Both sellers supply a homogeneous product, with zero production costs without loss of generality. Although consumers can only be located within the arc of circle $[v, 1]$, they can travel along the whole circle and will always take the direction that implies the shorter distance to the chosen firm. The distance between the consumer's location $\alpha$ and the firm's location $x_{i}$ is given by $d_{i}=\left|\alpha-x_{i}\right|, i=1,2$. Consumers are evenly distributed along the residential area $[v, 1]$, and buy a single unit of product.

Since the product is homogeneous, consumers will buy from the firm offering the lowest total price, that is, mill price plus transportation costs. A linear-quadratic transport cost function which can be either convex or concave is considered.

The timing of the game is the following: in the first stage firms choose locations and in the second stage firms compete in prices. The concept used to solve the model is perfect equilibrium; the game is then solved by backward induction.

The questions that arise in this context are:

(i) Are the convex case and the concave case related via a change of parameters?

(ii) Is there price equilibrium for any locations $x_{1}, x_{2}$ in the circular market with zoning?

\subsection{Demand function}

The indifferent consumers determine the market boundaries between the two firms so that demand can be derived. Again, a consumer who faces the same full price (mill price plus transport cost) from the two firms is labeled as the indifferent consumer. Depending on the price and the location of firm, it is possible that an indifferent consumer exists in regions $\left[v, x_{1}+1 / 2\right],\left[x_{1}+1 / 2, x_{2}+1 / 2\right],\left[x_{2}+1 / 2,1\right]$ on the circle.

Under a convex linear quadratic transport cost function, such as, $C^{+}\left(d_{i}\right)=a^{+} d_{i}+b^{+} d_{i}^{2}$, where $a^{+} \geq 0, b^{+} \geq 0$, if there exists an indifferent consumer $^{4}$, she/he must be necessarily located in the region $\left[x_{1}+1 / 2, x_{2}+1 / 2\right]^{5}$. Let $\alpha_{B}^{+}$be the location of this indifferent consumer. In this case, consumers to the right of $\alpha_{B}^{+}$prefer seller 1 , while consumers to the left of $\alpha_{B}^{+}$prefer seller 2 . Furthermore, there can be a second indifferent consumer in either $\left[v, x_{1}+1 / 2\right]$ or $\left[x_{2}+1 / 2,1\right]$ denoted $\alpha_{A}^{+}$or $\alpha_{c}^{+}$.

Substituting the expression for the location of the indifferent consumer, the demand of firm 1 is then given by:

\footnotetext{
The expression of indifferent consumer is given by: $\mathrm{p} 1+\mathrm{C}(\mathrm{d} 1)=\mathrm{p} 2+\mathrm{C}(\mathrm{d} 2)$

5 See De Frutos et al. (2002).
} 


$$
D_{1}^{+}\left(v, x_{1}, x_{2}, p_{1}, p_{2}\right)= \begin{cases}(1-v) & \text { if } p_{1}-p_{2} \in I_{1}^{+} \\ \left(\frac{\left(a^{+}+b^{+}\right)\left(p_{2}-p_{1}\right)}{2 b^{+} z\left[a^{+}+b^{+}-b^{+} z\right]}-\frac{a^{+}}{2 b^{+}}+\frac{1}{2}-v\right) & \text { if } p_{1}-p_{2} \in I_{2}^{+} \\ \left(\frac{p_{2}-p_{1}}{2\left[a^{+}+b^{+}-b^{+} z\right]}-\frac{q}{2}+\frac{1}{2}\right) & \text { if } p_{1}-p_{2} \in I_{3}^{+} \\ \left(\frac{\left(a^{+}+b^{+}\right)\left(p_{2}-p_{1}\right)}{2 b^{+} z\left[a^{+}+b^{+}-b^{+} z\right]}+\frac{a^{+}}{2 b^{+}}+\frac{1}{2}\right) & \text { if } p_{1}-p_{2} \in I_{4}^{+} \\ 0 & \text { if } p_{1}-p_{2} \in I_{5}^{+}\end{cases}
$$

where $z=x_{2}-x_{1}, q=\left(x_{2}+x_{1}\right) / 2$ :

$I_{1}^{+}=\left[-\infty,-z\left(a^{+}+b^{+}-b^{+} z\right)\right]$,

$I_{3}^{+}=\left[-z\left(a^{+}+2 b^{+} v-b^{+} q\right), z\left(a^{+}+b^{+} q\right)\right]$,

$I_{5}^{+}=\left[z\left(a^{+}+b^{+}-b^{+} z\right),+\infty\right]$.

When the transport cost function is concave, and given by $C^{-}\left(d_{i}\right)=a^{-} d_{i}-b^{-} d_{i}^{2}$, where $a^{-} \geq b^{-} \geq 0^{6}$; no more than one indifferent consumer, $\alpha_{A}^{-}$or $\alpha_{B}^{-}$or $\alpha_{c}^{-}$, can exist located in the following intervals: $\left[v, x_{1}+1 / 2\right],\left[x_{1}+1 / 2, x_{2}+1 / 2\right]$, $\left[x_{2}+1 / 2,1\right]$. The demand function $D_{1}^{-}$is then represented by:

$$
D_{1}^{-}\left(v, x_{1}, x_{2}, p_{1}, p_{2}\right)= \begin{cases}(1-v) & \text { if } p_{1}-p_{2} \in I_{1}^{-} \\ \left(\frac{\left(p_{2}-p_{1}\right)}{2 b^{-} z}-\frac{q}{2}+1-\frac{a^{-}}{2 b^{-}}\right) & \text {if } p_{1}-p_{2} \in I_{2}^{-} \\ \left(\frac{p_{2}-p_{1}}{2\left[a^{-}-b^{-}+b^{-} z\right]}-\frac{q}{2}+\frac{1}{2}\right) & \text { if } p_{1}-p_{2} \in I_{3}^{-} \\ \left(\frac{\left(p_{2}-p_{1}\right)}{2 b^{-} z}-\frac{q}{2}+\frac{a^{-}}{2 b^{-}}\right) & \text {if } p_{1}-p_{2} \in I_{4}^{-} \\ 0 & \text { if } p_{1}-p_{2} \in I_{5}^{-}\end{cases}
$$

where $z=x_{2}-x_{1}, q=\left(x_{2}+x_{1}\right) / 2$ :

$$
\begin{aligned}
& I_{1}^{-}=\left[-\infty,-z\left(a^{-}-2 b^{-} v+b^{-} q\right)\right], \quad I_{2}^{-}=\left[-z\left(a^{-}-2 b^{-} v+b^{-} q\right),-z\left(a^{-}-b^{-}+b^{-} z\right)\right] \\
& I_{3}^{-}=\left[-z\left(a^{-}-b^{-}+b^{-} z\right), z\left(a^{-}-b^{-}+b^{-} z\right)\right], \quad I_{4}^{-}=\left[-z\left(a^{-}-b^{-}+b^{-} z\right), z\left(a^{-}+b^{-} q\right)\right] \\
& I_{5}^{-}=\left[z\left(a^{-}+b^{-} q\right),+\infty\right] .
\end{aligned}
$$

$\overline{6} a^{-} \geq b^{-}$is assumed to guarantee increasing transport costs in all the market. 
Let $G\left(C, p_{1}, p_{2}, x_{1}, x_{2}\right)$, represent the game induced by the transport cost function $\mathrm{C}$ and by the pair of locations $\left(x_{1}, x_{2}\right)$, where $C=C^{+}$or $C=C^{-}$.

\section{Proposition 2}

The two price subgames $G\left(C^{+}, p_{1}, p_{2}, x_{1}, x_{2}\right), G\left(C^{-}, p_{1}, p_{2}, x_{1}, x_{2}\right)$ are not equivalent.

\section{Proof:}

Comparing the above demand $D^{+}\left(v, p_{1}, p_{2}, x_{1}, x_{2}\right)$ and $D^{-}\left(v, p_{1}, p_{2}, x_{1}, x_{2}\right)$, it is easy to see that by using the appropriate transformation, $C^{-}(d)=C^{+}(1 / 2)-C^{+}(1 / 2-d)$ i.e. $a^{+}=a^{-}-2 b^{-} L, b^{+}=b^{-}$, the demand for the convex case $D^{+}$and the demand for the concave case $D^{-}$are different. Therefore, taking into the definition of equivalent games ${ }^{7}$, it can be deduced that both price subgames $G\left(C^{+}, p_{1}, p_{2}, x_{1}, x_{2}\right), G\left(C^{-}, p_{1}, p_{2}, x_{1}, x_{2}\right)$ are not equivalent.

\subsection{Nash equilibrium}

Starting from the second stage of the game, equilibrium in prices is discussed now. In this case, the commercial area $v$ and the locations of the enterprises $x_{1}, x_{2}$, are given. As with the original Hotelling approach, Bertrand competition is assumed. The profit of seller $i, \quad i=1,2$ is denoted by $B_{I}\left(p_{i}, p_{j}\right)=p_{i} D_{I}\left(p_{i}, p_{j}\right), \quad i \neq j$. From

$$
\underset{p_{i}}{\operatorname{Max}} B_{I}\left(p_{i}, p_{j}\right) \quad i=1,2
$$

Subsequently, the first conditions are :

$$
\frac{\partial B_{I}\left(p_{i}, p_{j}\right)}{\partial p_{i}}=D_{I}+p_{i} \frac{\partial D_{I}\left(p_{i}, p_{j}\right)}{\partial p_{i}}=0, \quad i=1,2
$$

By solving the conditions for both firms simultaneously, Nash equilibrium in price strategies can be obtained. Then, the following proposition is stated for the convex case:

\section{Proposition 3:}

Assuming the transport cost as the quadratic lineal convex function, $C^{+}(d)=a^{+} d+b^{+} d^{2}, \quad a^{+} \geq 0, b^{+}>0$, there is not a Nash price equilibrium for any pair of locations, $\left(x_{1}, x_{2}\right) \in[0, v]$ such that $0 \leq v \leq 1 / 2$.

$7 \quad$ See De Frutos et al. (2002, definition1 pp. 535): Two price subgames G (F, x1, x2) and $\mathrm{G}^{\prime}\left(\mathrm{F}^{\prime}, \mathrm{x} 1\right.$ ', $\mathrm{x} 2$ ') are full equivalent iff for every firm I, and for any strategy profile $\mathrm{p} \in \mathrm{R}^{2}$. 
Proof: (see Appendix)

Remarks:

The failure of the existence of Nash price equilibrium with convex and strictly linear-quadratic transport cost $\left(a^{+}>0, b^{+}>0\right)$ is expected given that a similar result is obtained in the linear and circular market. More surprisingly ${ }^{8}$, under quadratic transport costs, $\left(a^{+}=0\right)$ price equilibrium is not found for any location pair.

Intuitively, the non existence of Nash price equilibrium with the quadratic transport cost is justified by the reduction of the space for consumer's location. This pushes firms to compete through prices in a more intensive way. Furthermore, the average distance that consumers are covering increases once the short arc of the circumference behind firms is removed and not considered anymore as residential space. As the average distance is longer, the average transport cost increases more than proportionally and thus every firm is tempted to reduce prices trying to attract the highest number of consumers so that equilibrium ceases to exist. The reason is that a key equilibrium property does not hold in this context i.e no firm can increase its profit through a unilateral change in price.

\section{Proposition 4:}

Considering a linear quadratic concave transport cost function, $C^{-}(d)=a^{-} d-b^{-} d^{2}$, such that $a^{-} \geq b^{-}>0$ :

i) If $a^{-}>b^{-}>0$, there is not price equilibrium for any location pair $\left(x_{1}, x_{2}\right) \in[0, v]$.

ii) If $a^{-}=b^{-}$, i.e. the transport cost is represented as $C^{-}(d)=a^{-}\left(d-d^{2}\right)$, there exists a subgame perfect price-location equilibrium. Therefore, the equilibrium is unique and given by: $x_{1}^{*}(v)=0, x_{2}^{*}(v)=v, p_{1}^{*}\left(x_{1}^{*}, x_{2}^{*}\right)=p_{2}^{*}\left(x_{1}^{*}, x_{2}^{*}\right)=b^{-} v(1-v)$.

\section{Proof: (see Appendix)}

\section{Remarks:}

Prices are increasing with respect to $v$ in perfect equilibrium. Thus, a large value for the shopping area, $v$, might involve less price competition. Consequently, firms prefer to separate from each other within the retail area leading to maximum differentiation.

Hamoudi et al. (2012) have analysed a concave linear quadratic transport cost for the particular case in which $a^{-}=b^{-} \geq 0$, with the aim of finding out the optimal size of commercial area $[0, v]$. For $v=1 / 2$ and an identical function, the same result is found as in De Frutos et al. (1999), namely:

8 In the linear and circular market there exist equilibrium under quadratic transport cost (Gabszewicz et al. (1986), Anderson (1988), De Frutos et al. (1999), Hamoudi et al. (2011)). 
$x_{1}^{*}=0, x_{2}^{*}=1 / 2, p_{1}^{*}=p_{2}^{*}=b^{-} / 4$ Similarly, this case corresponds to spatial dispersion/maximum differentiation.

An intuitive explanation is given by the fact that when separating the commercial and residential areas, the average distance increases; however, it does so differently with the concave transport cost function and the convex one. Thus, the weight of the transport cost is not important enough to induce fierce price competition.

\section{Conclusions}

In this article the problem of the equivalence between a quadratic lineal convex cost of transport and a concave one is initially reconsidered in the model of spatial competition in a circular city. The length of space is assumed arbitrary instead of unitary. The result obtained is that the relation of equivalence depends on the perimeter of the circular city.

When convex and concave linear quadratic transport cost functions are considered an equivalence relationship is obtained. This relationship is a key result in theoretical and practical terms. On one hand, it shows a link between the weights attached to the linear and non linear parts of the transport cost functions and the length of the circular market considered. On the other hand, it can be taken as a reference by any urban city planner interested in choosing the size of the market. A regulator can consider a priori criteria on the weight of the parameters in the transport cost functions when deciding on the ideal size of the space market; given that once equilibrium is reached for a convex/concave function it is also ensured for the concave/convex case. More importantly, the result shown allows a city planner to be indifferent about the type of transport cost function. This is a remarkable contribution as considerations on the type of transport cost function are often due to technical reasons and lack economic foundation.

Secondly, a regulated circular space is considered in which a planner decides about the spatial configuration where firms and consumers are forced to locate in different areas of the circle. There is not equivalence in the games induced by a convex lineal-quadratic transport cost function and a concave function.

On the other hand, the convex or concave strictly linear quadratic transport cost function involves similar equilibrium problems to those found in the standard circular or linear model. Furthermore, even for the particular case of a convex quadratic transport cost, the equilibrium failure persists. This is a rather surprising result since the quadratic transport cost function has always guaranteed equilibrium existence in spatial competition models.

Nonetheless, a concave quadratic transport cost function is found, that re-establishes the Nash equilibrium in prices for any location of firms. In this location equilibrium firms locate at the end of the commercial area, corresponding to maximum product differentiation/spatial dispersion.

Finally, the striking fact is the failure of equilibrium with the convex quadratic transport cost when it exists under concave quadratic transport cost. This results stands in sharp contrast to those stemming under a linear market assumption. It is the type of zoning regulation in this model which creates the nonexistence 
problem of equilibrium with convex quadratic transport cost. Indeed, it is rather simple to prove that price equilibrium exists for any locations with convex or concave quadratic costs when consumers are allowed to locate in the commercial area from the circular or linear market

\section{REFERENCES}

Anderson, S.P. (1986). "Equilibrium Existence in The Circle Model of Product Differentiation", Papers in Regional Science, Series16 ; 19-29.

Anderson, S.P. (1988). "Equilibrium Existence in The Linear Model of Spatial Competition”, Economica 55; 479-491.

Böckem, S. (1994). "A generalized Model of Horizontal Product Differentiation", The Journal of Industrial Economics 42; 287-298.

Brenner, S. (2005). "Hotelling Game with Three, Four, and More Players", Journal of Regional Science 45; 851-864.

Carpenter, Ch. (2007). "Heavy Alcohol Use and Crime: Evidence from Underage Drunk-Driving Laws", Journal of Law and Economics 50 (3); 539-557.

Cook, P. et al. (2000). "Alcohol", In Handbook of Health Economics, in: Culyer $\&$ Newhouse (ed), Handbook of Health Economics edition 1, V 1, chap. 30; 1629-1673 Elsevier.

D’Aspremont, et al. (1979). “On Hotelling.s stability in competition”, Econometrica 47; 1145-1150.

De Frutos M.A. et al. (1999). "Equilibrium existence in the circle model with linear quadratic transport costs". Regional Science \& Urban Economics 29; 605-615.

De Frutos M.A. et al. (2002). "Spatial Competition with concave Transport costs", Regional Science \& Urban Economics, 32; 531-540.

Gabszewicz, et al. (1986). "On the nature of competition with differentiated products", The Economic Journal 96; 160-172.

Hamoudi, H. et al. (2005). "Equilibrium Existence in the Linear Model: Concave Versus Convex Transport", Papers in Regional Science 84; 201-219.

Hamoudi, H. et al. (2011). "Revisiting price equilibrium in the linear city model of spatial competition”. Papers in Regional Science 90; 179-196.

Hamoudi, H. et al. (2012). "The Effect of Zoning in Spatial Competition". Journal of Regional Science 52; 361-374.

Hotelling, H. (1929). “Stability in Competition”. The Economic Journal 39; 41-57. Junichiro, I. et al. (2004). "A non-cooperative analysis of a circular city model", Regional Science \&Urban Economics 34; 575-589.

Lambertini, L. (1994). "Equilibrium Location in the Unconstrained Hotelling Game", Economic Notes 24; 438-446.

Matsumura, T. et al. (2006). "A Note on the Excess Entry Theorem in Spatial Markets", International Journal of Industrial Organization 24; 1071-1076.

Netz, J. et al. (2002). "Maximum or Minimum Differentiation? Location Patterns of Retail Outlets", The Review of Economics and Statistics, 84 (1); 162-175.

Tabuchi, T. et al. (1995). "Asymmetric equilibria in Spatial Competition". International Journal of industrial Organization 13; 213-227. 


\section{Appendix}

\section{Proof of Proposition 3:}

Given the definition of Nash's equilibrium in prices and the expressions of the profit functions, the solution can be calculated easily by using the first order condition:

Case 1: Equilibrium prices in region $\mathrm{I}_{2}^{+}$.

$$
\begin{aligned}
& \left(\partial B_{1}^{+} / \partial p_{1}\right)=0 \Leftrightarrow \quad\left(a^{+}+b^{+}\right)\left(p_{2}-2 p_{1}\right)+z\left(a^{+}+b^{+}-b^{+} z\right)\left(b^{+}-2 b^{+} v-a\right)=0 \\
& \left(\partial B_{2}^{+} / \partial p_{2}\right)=0 \Leftrightarrow \quad\left(a^{+}+b^{+}\right)\left(p_{1}-2 p_{2}\right)+z\left(a^{+}+b^{+}\right)\left(a^{+}+b^{+}-b^{+} z\right)=0
\end{aligned}
$$

By solving the two previous equations simultaneously the result is given by:

$$
p_{1}^{*}=\frac{z\left(a^{+}+b^{+}-b^{+} z\right)}{3\left(a^{+}+b^{+}\right)}\left[b^{+}(3-4 v)-a^{+}\right] p_{2}^{*}=\frac{z\left(a^{+}+b^{+}-b^{+} z\right)}{3\left(a^{+}+b^{+}\right)}\left[b^{+}(3-2 v)+a^{+}\right]
$$

This solution must also verify the condition $p_{1}^{*}(z, q, v)-p_{2}^{*}(z, q, v) \in I_{2}^{+}$

i) $-z\left(a^{+}+b^{+}-b^{+} z\right) \leq p_{1}^{*}-p_{2}^{*} \Leftrightarrow 3\left(a^{+}+b^{+}\right)-2\left(b^{+} v+a\right) \geq 0$,

This inequality always holds.

ii) $p_{1}^{*}-p_{2}^{*} \leq-z\left(a^{+}+2 b^{+} v-b^{+} q\right)$

$\Leftrightarrow\left(b^{+}\right)^{2}(3 q-2 z v)+a^{+} b^{+}(3 q-2 z)-\left(a^{+}+b^{+}\right)\left(a^{+}+4 b^{+2} v\right) \geq 0$.

However, this inequality does not hold for every value of $\mathrm{z}, \mathrm{q}, \mathrm{a}, \mathrm{b}, \mathrm{v}$. Therefore equilibrium in prices will exist if the previous condition is fulfilled, so it can be stated that there is not a equilibrium in prices in region $I_{2}^{+}$for every value of $\mathrm{z}$ and $\mathrm{q}$.

\section{Case 2: Equilibrium prices in region $I_{3}^{+}$.}

$\left(\partial B_{1}^{+} / \partial p_{1}\right)=0$ is equivalent to: $\left(p_{2}-2 p_{1}\right)+\left(a^{+}+b^{+}-b^{+} z\right)(1-q)=0$

$\left(\partial B_{2}^{+} / \partial p_{2}\right)=0$ is equivalent to: $\left(p_{1}-2 p_{2}\right)+\left(a^{+}+b^{+}-b^{+} z\right)(1+q-2 v)=0$.

The equilibrium prices are obtained by solving the two previous equations together and so that their expressions will be given by:

$$
p_{1}^{* * *}=(z / 3)\left(a^{+}+b^{+}-b^{+} z\right)(3-q-2 v), \quad p_{2}^{* * *}=(z / 3)\left(a^{+}+b^{+}-b^{+} z\right)(3+q-4 v) .
$$


Nevertheless, this solution has to verify the condition $p_{1}^{* *}(z, q, v)-p_{2}^{* *}(z, q, v) \in I_{3}^{+}$
$i)-z\left(a^{+}+2 b^{+} v-b^{+} q\right) \leq p_{1}^{* *}-p_{2}^{* *} \Leftrightarrow b^{+} z q-z\left(3 a^{+}+4 b^{+} v\right)+2\left(a^{+}+b^{+}\right)(q-v) \leq 0$
ii) $p_{1}^{* *}-p_{2}^{* *} \leq z\left(a^{+}+b^{+} q\right) \Leftrightarrow b^{+} z q+2 b^{+} q+2 b^{+} v z-2\left(a^{+}+b^{+}\right) v \geq 0$.

However, these inequalities do not hold for every value of $\mathrm{z}, \mathrm{q}, \mathrm{a}, \mathrm{b}, \mathrm{v}$. Consequently, equilibrium in prices will exist if the previous condition is carried out so it can be assured that there is no equilibrium in prices in region $I_{3}^{+}$ for every value of $z$ and $q$.

\section{Case 3: Equlibrium prices in region $I_{4}$.}

$\left(\partial B_{1}^{+} / \partial p_{1}\right)=0$ is equivalent to: $\left(a^{+}+b^{+}\right)\left(p_{2}-2 p_{1}\right)+z\left(a^{+}+b^{+}\right)\left(a^{+}+b^{+}-2 b^{+} z\right)=0$

$\left(\partial B_{2}^{+} / \partial p_{2}\right)=0 \Leftrightarrow\left(a^{+}+b^{+}\right)\left(p_{1}-2 p_{2}\right)+z\left(a^{+}-2 b^{+} v-a^{+}\right)\left(a^{+}+b^{+}-b^{+} z\right)=0$

The equilibrium prices are derived by solving the two previous equations:

$$
p_{1}^{* * *}=(z / 3)\left(a^{+}+b^{+}-b^{+} z\right)\left[b^{+}(3-2 v)+a^{+}\right], p_{2}^{* * *}=(z / 3)\left(a^{+}+b^{+}-b^{+} z\right)\left[b^{+}(3-4 v)-a^{+}\right]
$$

Nonetheless, it has to be verified that $p_{1}^{* * *}(z, q, v)-p_{2}^{* * *}(z, q, v) \in I_{4}^{+}$,

i) $z\left(a^{+}+b^{+} q\right) \leq p_{1}^{* * *}-p_{2}^{* * *}$

$\Leftrightarrow b^{+2}(3 q-2 z v)+a^{+} b^{+}(3 q-2 z)+\left(a^{+}+b^{+}\right)\left(a^{+}-2 b^{+2} v\right) \geq 0$.

However, this inequality does not hold for every value of $\mathrm{z}, \mathrm{q}, \mathrm{a}, \mathrm{b}, v$.

ii) $p_{1}^{* * *}-p_{2}^{* * *} \leq z\left(a^{+}+b^{+}-b^{+} z\right)$

This inequality is always carried out. Therefore there will be price equilibrium if the previous condition holds in which case it can be asserted that there is not equilibrium in prices in region $I_{4}$ for any value of $\mathrm{z}$ and $\mathrm{q}$.

\section{Proof of Proposition 4:}

The equilibrium prices can be calculated by using the first order condition:

Case 1: Equilibrium prices in region $\mathrm{I}_{2}^{-}$.

$\left(\partial B_{1}^{-} / \partial p_{1}\right)=0$ is equivalent to: $\left(p_{2}-2 p_{1}\right)+z\left(2 b^{-}-b^{-} q-a^{-}\right)=0$

$\left(\partial B_{2}^{-} / \partial p_{2}\right)=0$, is equivalent to: $\left(p_{1}-2 p_{2}\right)+z\left(a^{-}+b^{-} q-2 b^{-} v\right)=0$.

Equilibrium prices are obtained by solving the two previous equations, their expressions are given by: 


$$
p_{1}^{*}=(z / 3)\left[2 b^{-}(2-v)-\left(a^{-}+b^{-} q\right)\right], \quad p_{2}^{*}=(z / 3)\left[2 b^{-}(1-2 v)+\left(a^{-}+b^{-} q\right)\right]
$$

However this solution has to verify the condition $p_{1}^{*}(z, q, v)-p_{2}^{*}(z, q, v) \in I_{2}^{-}$

$$
\text { i) }-z\left(a^{-}-2 b^{-} v-b^{-} q\right) \leq p_{1}^{*}-p_{2}^{*} \Leftrightarrow 2 b^{-}(1-2 v)+\left(a^{-}+b^{-} q\right) \geq 0
$$

This inequality is always fulfilled.

ii) $p_{1}^{*}-p_{2}^{*} \leq-z\left(a^{-}+2 b^{-} v-b^{-} q\right) \Leftrightarrow\left(a^{-}+b^{-} q\right)-b^{-}(1-2 v) \leq 0$.

This inequality does not hold for every value of $\mathrm{z}, \mathrm{q}, \mathrm{a}, \mathrm{b}, v$ and $a^{-} \neq b^{-}$. However, both inequalities can be verified for any value of $\mathrm{z}, \mathrm{q}, \mathrm{v}$ and $a^{-}=b^{-}$. It can then be guaranteed that no equilibrium in prices in region $I_{3}^{-}$exists for every value of $\mathrm{z}$ and $\mathrm{q}$ and $a^{-} \neq b^{-}$. Nevertheless, equilibrium exists if $a^{-}=b^{-}$

\section{Case 2: Equilibrium prices in region $\mathrm{I}_{3}^{-}$.}

$\left(\partial B_{1}^{-} / \partial p_{1}\right)=0$, is equivalent to: $\left(p_{2}-2 p_{1}\right)+(1-q)\left(a^{-}-b^{-}+b^{-} z\right)=0$ $\left(\partial B_{2}^{-} / \partial p_{2}\right)=0$ is equivalent to: $\left(p_{1}-2 p_{2}\right)+z\left(a^{-}+b^{-} q-2 b^{-} v\right)=0$.

The equilibrium prices are obtained by solving the two previous equations:

$$
p_{1}^{* *}=(1 / 3)\left(a^{-}-b^{-}+b^{-} z\right)(3-q-2 v), \quad p_{2}^{* *}=(1 / 3)\left(a^{-}-b^{-}+b^{-} z\right)(3+q-4 v)
$$

Nevertheless this solution has to verify the condition $p_{1}^{* *}(z, q, v)-p_{2}^{* *}(z, q, v) \in I_{3}^{-}$:

$$
\begin{aligned}
& \text { i) }-z\left(a^{-}-b^{-}+b^{-} z\right) \leq p_{1}^{* *}-p_{2}^{* *} \Leftrightarrow 2 q-3 z-2 v \leq 0 \\
& \text { ii) } p_{1}^{* *}-p_{2}^{* *} \leq z\left(a^{-}+b^{-}-b^{-} z\right) \Leftrightarrow 2 q+3 z-2 v \geq 0 .
\end{aligned}
$$

These inequalities do not hold for every value of $\mathrm{z}, \mathrm{q}$, and $v$ and $a^{-} \neq b^{-}$.

However, both inequalities can be verified for any value of $\mathrm{z}, \mathrm{q}, \mathrm{v}$ and $a^{-}=b^{-}$

\section{Case 3: Equilibrium prices in region $I_{4}^{-}$.}

$\left(\partial B_{1}^{-} / \partial p_{1}\right)=0$ is equivalent to: $\left(p_{2}-2 p_{1}\right)+z\left(a^{-}-b^{-} q\right)=0$

$\left(\partial B_{2}^{-} / \partial p_{2}\right)=0$ is equivalent to: $\left(p_{1}-2 p_{2}\right)+z\left(2 b^{-}(1-v)-\left(a^{-}-b^{-} q\right)\right)=0$.

Equilibrium prices are obtained by solving the two previous equations and giving their expressions by:

$$
p_{1}^{* * *}=(z / 3)\left[2 b^{-}(1-v)+\left(a^{-}-b^{-} q\right)\right], \quad p_{2}^{* * *}=(z / 3)\left[4 b^{-}(1-v)-\left(a^{-}-b^{-} q\right)\right]
$$


Nonetheless this solution has to verify the condition $p_{1}^{* * *}(z, q, v)-p_{2}^{* * * *}(z, q, v) \in I_{4}^{-}$ i) $z\left(a^{-}-b^{-}+b^{-} z\right) \leq p_{1}^{* * *}-p_{2}^{* * *} \Leftrightarrow b^{-}(2 q+3 z)+\left(a^{-}-b^{-}\right)-2 b^{-} v \leq 0$ and ii) $\left.p_{1}^{* * *}-p_{2}^{* * *} \leq z\left(a^{-}-b^{-} q\right) \quad \Leftrightarrow \quad\left(-b^{-} q\right)+\left(a^{-}-b^{-}\right)+2 b^{-} v\right) \geq 0$.

These inequalities do not hold for every value of $\mathrm{z}, \mathrm{q}, \mathrm{a}, \mathrm{b}$ and $v$ and $a^{-} \neq b^{-}$

It can then be guaranteed that no equilibrium in prices in region $I_{3}^{-}$exists for every value of $\mathrm{z}$ and $\mathrm{q}$ and $a^{-} \neq b^{-}$. Nevertheless, equilibrium exists if $a^{-}=b^{-}$

If $a^{-}=b^{-}$then, price equilibrium exists. By substituting these equilibrium prices in the profit functions, simple calculation show that location equilibrium exists and is given by: $x_{1}^{*}(v)=0, x_{2}^{*}(v)=v$. Therefore, corresponding prices are: $p_{1}^{*}=p_{2}^{*}=b^{-} v(1-v)$. 